\title{
Galaxies driven only by secular evolution?
}

\author{
Lourdes Verdes-Montenegro on behalf of the AMIGA team
}

Instituto de Astrofisica de Andalucia (CSIC), email: lourdes@iaa.es

\begin{abstract}
The AMIGA project (Analysis of the interstellar Medium of Isolated GAlaxies, http://amiga.iaa.es) has identified a significant sample of very isolated ( $T_{c c}$ (nearest-neighbor) $\approx 2-3 \mathrm{Gyr}$ ) galaxies in the local Universe and revealed that they have different properties than galaxies in richer environments. Our analysis of a multiwavelength database includes quantification of degree of isolation, morphologies, as well as FIR and radio line/continuum properties.

Properties usually regarded as susceptible to interaction enhancement show lower averages in AMIGA-lower than any galaxy sample yet identified. We find lower MIR/FIR measures (Lisenfeld et al. 2007), low levels of radio continuum emission (Leon et al. 2008), no radioexcess above the radioFIR correlation (0\%, Sabater et al.2008), a small number of AGN (22\%, Sabater et al. 2012), and lower molecular gas content (Lisenfeld et al. 2011). The late-type spiral majority in our sample show very small bulge/total ratios (largely $<0.1$ ) and Sersic indices consistent with an absence of classical bulges (Durbala et al. 2008). They show redder $g-r$ colors and lower color dispersion for AMIGA subtypes (Fernandez-Lorenzo et al. 2012) and show the narrowest (gaussian) distribution of HI profile asymmetries of any sample yet studied.

This work has been supported by Grant AYA2011-30491-C02-01 co-financed by MICINN and FEDER funds, and the Junta de Andalucia (Spain) grants P08-FQM-4205 and TIC-114.
\end{abstract}

\section{References}

Durbala, A., et al. 2008, MNRAS 390, 881

Fernandez Lorenzo, M., et al. 2012, A\&A 540, 47

Leon, S., et al. 2008, A\&SA 485, 475

Lisenfeld, U., et al. 2007, A\&A 462, 507

Lisenfeld, U., et al. 2011, A\&A 534, 102

Sabater, J., et al. 2008, A\&SA 486, 73

Sabater, J., et al. 2012, A\&SA 545, 15 